\title{
Immunochemical analysis of iron transporters and M2 macrophages in ovarian endometrioma and clear cell adenocarcinoma
}

\author{
KEIKO AKASHI $^{1}$, YOJI NAGASHIMA ${ }^{2}$, TSUTOMU TABATA $^{3}$ and HIDEAKI ODA ${ }^{1}$ \\ Departments of ${ }^{1}$ Pathology, ${ }^{2}$ Surgical Pathology and ${ }^{3}$ Obstetrics and Gynecology, \\ Tokyo Women's Medical University, Shinjuku, Tokyo 162-8666, Japan
}

Received January 11, 2021; Accepted May 21, 2021

DOI: $10.3892 / \mathrm{mco} .2021 .2321$

\begin{abstract}
The association between iron ions and endometriosis-associated ovarian cancer (EAOC) has been previously investigated to elucidate EAOC carcinogenesis; however, the dynamics of iron deposition in the endometrial epithelium and endometrial stroma of ovarian endometrioma $(\mathrm{OE})$ remains unknown. The present study aimed to determine the expression of iron transporters on the cell surface and the distribution of tumor-associated macrophages (TAMs) englobed with iron in the endometrial stroma. The current retrospective study investigated $20 \mathrm{OE}$ and 18 ovarian clear cell adenocarcinoma (CCC) samples, using Perls Prussian blue staining and immunohistochemistry of iron transporters, including divalent metal transporter 1 (DMT1), transferrin receptor (TfR) and ferroportin (FPN). Additionally, samples were stained for CD68, CD11c, CD163 and CD206, and double-immunostained for iron and CD163 to define the distribution of macrophages. Iron transporters were identified on the endometrial epithelium of $\mathrm{OE}$ and $\mathrm{CCC}$ tumor cells, and TAMs were englobed with iron in the endometrial stroma of OE and CCC. Histological findings revealed DMT1 upregulation in OE and CCC, whereas lower TfR and FPN expression was observed in OE than in CCC. M2 macrophages were englobed with iron ions in the deep layers of the OE and CCC stroma. The endometrial epithelium located in the endometrial stroma of one patient with $\mathrm{OE}$ and the endometrial epithelium adjacent to $\mathrm{CCC}$ in
\end{abstract}

Correspondence to: Dr Keiko Akashi, Department of Pathology, Tokyo Women's Medical University, 8-1 Kawada-Cho, Shinjuku, Tokyo 162-8666, Japan

E-mail: ogu.kuma@gmail.com

Abbreviations: CCC, clear cell adenocarcinoma; DMT1, divalent metal transporter 1; EAOC, endometriosis-associated ovarian cancer; FPN, ferroportin; OE, ovarian endometrioma; TAMs, tumor-associated macrophages; TfR, transferrin receptor; H\&E staining, hematoxylin-eosin staining

Key words: OE, EAOC, ovarian CCC, iron transporter, TAM two patients with CCC stained positive for the tumor proliferation marker Ki67. Epithelium infiltrating the stroma of OE may become the origin of cancer under the influence of M2 macrophages englobed with iron. These findings provide new perspectives on the malignant transformation of $\mathrm{OE}$ into EAOC and its possibility as a precancerous index.

\section{Introduction}

Endometriosis is a pathology of the female reproductive tract (1), with a morbidity rate of $\sim 10 \%$ in women of reproductive age (2). Ovarian endometriomas (OE) are clinical manifestations of endometriosis and comprise a risk factor for the development of ovarian clear cell adenocarcinoma (CCC) and endometrioid carcinoma, regarded as endometriosis-associated ovarian cancers (EAOC) $(3,4)$. The primary histological type of EAOC in Asia is CCC, accounting for $20 \%$ of all ovarian cancers (5). The prevalence of EAOC will likely increase along with that of endometriosis; however, the underlying mechanisms associated with carcinogenesis are controversial. Hence, it is important to elucidate the mechanisms underlying malignant transformation and pathogenesis of EAOC.

Endometriosis is caused due to the reflux of menstrual blood containing endometrial tissues (6), and OE form when menstrual blood flows into the ovary (6), carrying endometrial epithelium and endometrial stroma into the ovary (7). Moreover, iron within the menstrual blood serves as an etiological factor for the development of ovarian cancer from OE (8-10). However, the precise mechanism through which iron ions flow in endometrial epithelial cells remains unknown.

Iron ions are primarily absorbed by the epithelium of the intestinal tract (11), after which iron is transported by divalent metal transporterl (DMT1) that imports and ferroportin (FPN) that exports iron ions $(12,13)$. Furthermore, transferrin receptors (TfR) uptake iron ions in other organs (14). These transporters regulate intracellular iron concentrations (11). Meanwhile, iron transporters in $\mathrm{OE}$ and $\mathrm{CCC}$ have rarely been investigated, and the mechanism of iron flow through endometrial epithelial cells of OE or CCC tumor cells remains unclear.

Macrophages within the endometrial stroma of $\mathrm{OE}$ accumulate around the epithelium to englobe iron (7). The 
M1 macrophage phenotype induces inflammatory cytokines and bactericidal activity (15), and M2 macrophages polarize toward tumor-associated macrophages (TAMs) after $\mathrm{T}$ cells and tumor cells release cytokines such as IL-4, IL-13, and macrophage colony-stimulating factor (M-CSF) (15). Thereafter, TAMs migrate to hypoxic regions, where they release growth factors and angiogenetic factors that promote proliferation and metastasis (16). Although M2 macrophages have been detected in OE and CCC $(17,18)$, their effect on OE carcinogenesis has not been investigated.

The present study aimed to immunohistochemically determine the expression of iron transporters in the endometrial epithelium of OE and tumor cells of CCC. We also evaluated the relation of macrophages to endometrial epithelial carcinogenesis in $\mathrm{OE}$.

\section{Materials and methods}

Samples. The study was approved by the Institutional Review Board of Tokyo Women's Medical University Hospital (Approval number 5371). We retrospectively analyzed $20 \mathrm{OE}$ tissues without malignant lesions and 18 ovarian CCC that were surgically excised at Tokyo Women's Medical University Hospital between January 2014 and December 2019. Opt-out informed consent was obtained from all patients.

Perls Prussian blue staining. Tissues were stained with Perls Prussian blue using potassium hexacyanoferrate (II) trihydrate (Wako Pure Chemical Industries, Osaka, Japan) and enhanced with Nuclear Fast Red (Cosmo Bio Co, Tokyo, Japan).

Hematoxylin and eosin (H\&E) staining and immunohistochemistry. Paraffin-embedded tissue sections $(4 \mu \mathrm{m})$ were deparaffinized for $10 \mathrm{~min}$. Then the sections were stained with $H \& E$ according to routine hospital procedures. For immunohistochemistry, antigen was retrieved by autoclaving for $20 \mathrm{~min}$ or microwave heating for $15 \mathrm{~min}$ in $10 \mathrm{mM}$ sodium citrate buffer, $\mathrm{pH} 6.0$ or 9.0. Nonspecific protein binding in tissue sections was blocked using Protein Block Serum-Free (Dako A/S, Glostrup, Denmark) at $25^{\circ} \mathrm{C}$, for $20 \mathrm{~min}$. The sections were incubated overnight at $4^{\circ} \mathrm{C}$ with primary antibodies, followed by secondary antibodies (Dako) for $30 \mathrm{~min}$ at $25^{\circ} \mathrm{C}$, and finally with $3,3^{\prime}$-diaminobenzidine (Nichirei Biosciences, Tokyo, Japan) to visualize antigen binding. Nuclei were stained with hematoxylin (Wako Pure Chemical Industries). Primary antibodies against the following were used: DMT1 (1:800), TfR (1:500), CD68 (1:400), CD11c (1:500), CD163 (1:500), and CD206 (1:5,000) (all from Abcam, Cambridge, United Kingdom), FPN (1:400; Novus Biologicals, Centennial, CO, USA), Ki67 (1:100) and cytokeratin (1:200) (both from Dako), and CD10 (1: 50; Leica Biosystems, Wetzlar, Germany). Samples prepared in the same manner but without incubation with the primary antibodies were used as negative controls (data not shown).

Immunohistochemistry analysis. The intensity of DMT1, TfR, and FPN staining was scored using an arbitrary scale as 0 , absent; 1, light; 2, moderate; 3 , intense. We used the score that was the most prevalent in 10 fields. Due to endometrial epithelial detachment, 10 fields could not be evaluated in three
OE samples, and hence, these samples were excluded from the analysis.

We evaluated CD11c, CD163, and CD206 expression as the average number of antibody-positive cells counted in 10 fields at $\mathrm{x} 400$ magnification. We evaluated ratios (\%) of Ki67-positive nuclei and averaged the numbers of cells counted at x400 magnification in 10 fields. Two pathologists assessed the immunohistochemical scores and numbers of positive cells in all sections.

Statistical analysis. Data were statistically analyzed using $\mathrm{JMP}^{\circledR} 14$ (SAS Institute Inc., Cary, NC, USA). Continuous variables are presented as mean \pm standard deviation. Categorical variables (Nulliparous and Premenopause) are presented as numbers (percentage) and compared with $\chi^{2}$ test or Fisher's exact test. Shapiro-Wilk test was performed to determine if the continuous variables were normally distributed. The levels of CA125 are expressed as the median with interquartile range (IQR) as they were not normally distributed. Comparison of two groups with normally distributed data (mean age and maximal cyst/tumor diameter) was performed using Welch's t-test; otherwise, comparison of continuous variables (median CA125) was performed using the non-parametric Wilcoxon rank sum test. Two groups and more than two groups were compared using the non-parametric Wilcoxon rank sum test and Steel-Dwass test (used after Kruskal-Wallis test), respectively, to confirm the significance of iron transport proteins and the number of positively immunostained cells. Statistical significance was set at $\mathrm{P}<0.05$.

\section{Results}

Clinical features. Table I lists the clinical features of the 18 CCC patients and $20 \mathrm{OE}$ patients. The average age of the CCC (56 years) and OE groups (47 years) differed significantly $(\mathrm{P}=0.0105)$. Most $\mathrm{CCC}$ patients had a stage I disease, according to the International Federation of Gynecology and Obstetrics (FIGO) staging guidelines. Of the 18 CCC patients, seven had a medical history of OE, two had a histopathological diagnosis of OE, one had both medical history and histopathological diagnosis of $\mathrm{OE}$, and the medical history of $\mathrm{OE}$ in eight patients was unknown. The maximal tumor diameter was significantly larger in $\mathrm{CCC}$ than that in $\mathrm{OE}(\mathrm{P}<0.001)$.

Iron deposition in $O E$ and $C C C$. We initially investigated iron localization in $\mathrm{OE}$ and $\mathrm{CCC}$ by staining tissue sections with Prussian blue (Fig. 1). Iron was deposited in the endometrial epithelium (Fig. 1A arrow) of four OE samples and englobed macrophages in the stroma just below the epithelium (Fig. 1A) of all OE samples. Iron was deposited throughout the stroma in all OE specimens (Table I). Stroma located beneath the aggregated englobed macrophages (referred to herein as 'deep layer'; Fig. 1B, region within the dotted line) also contained iron. Iron was deposited in CCC cancer cells and stroma in 13 specimens (Fig. 1C and Table I).

Immunostaining for iron transport proteins. Considering that iron deposition was observed in both OE and CCC, we investigated the expression of iron transport proteins DMT1, 
Table I. Characteristics of patients with CCC $(n=18)$ or OE $(n=20)$.

\begin{tabular}{|c|c|c|c|}
\hline Variable & $\mathrm{CCC}$ & $\mathrm{OE}$ & P-value \\
\hline Mean age (SD), years & $56(11)$ & $47(3)$ & 0.0105 \\
\hline Nulliparous, $\mathrm{n}(\%)$ & $9(50)$ & $13(65)$ & 0.5118 \\
\hline Premenopause, n (\%) & $5(27)$ & $19(95)$ & $<0.001$ \\
\hline Maximal cyst/tumor diameter, mean (SD), mm & $123(39)$ & $46(21)$ & $<0.001$ \\
\hline Median CA125 (IQR), U/ml & $45(21-184)$ & $58(28-172)$ & 0.6742 \\
\hline \multicolumn{4}{|l|}{ FIGO stage, n $(\%)$} \\
\hline I & $14(77)$ & & \\
\hline II & $1(5)$ & & \\
\hline III & $3(16)$ & & \\
\hline IV & $0(0)$ & & \\
\hline OE positive, $\mathrm{n}(\%)$ & $10(55)$ & & \\
\hline Iron deposition positive, $\mathrm{n}(\%)$ & $\begin{array}{l}13(72) \text { (in cancer } \\
\text { cells and stroma) }\end{array}$ & $\begin{array}{l}4(20) \text { (in endometrial epithelium) } \\
\text { and } 20 \text { (100) (throughout stroma) }\end{array}$ & \\
\hline
\end{tabular}

CCC, clear cell adenocarcinoma; OE, ovarian endometrioma; FIGO, International Federation of Gynecology and Obstetrics; IQR, interquartile range; CA125, cancer antigen 125 .

TfR, and FPN by immunostaining (Fig. 1D). All specimens of OE epithelium stained positive for these proteins. Specifically, DMT1 was scored 3 (intense staining) in 16 (Fig. 1E), TfR was scored 1 (light staining) in 12 (Fig. 1F), and FPN was scored 2 (moderate staining) in 11 (Fig. 1G) OE specimens. All three transporters were expressed at high levels in CCC. Comparing the expression of the iron transport proteins in $\mathrm{OE}$ and CCC specimens, we observed no remarkable difference in DMT1 expression (Fig. 1E), whereas expression of TfR and FPN was significantly higher $(\mathrm{P}<0.001)$ in $\mathrm{CCC}$ than in $\mathrm{OE}$ (Fig. 1F and G).

Macrophages with englobed iron infiltrate $O E$ and $C C C$. Fig. 1 indicates that iron ions flowed into and drained from cells. Iron was deposited not only in epithelial cells but also in the stroma of OE. Although Prussian blue staining revealed iron deposition in macrophages located immediately below the epithelium, it was difficult to ascertain whether iron ions in the entire stroma were englobed by macrophages. Hence, we examined the distribution of macrophages in the stroma to determine if they englobed iron ions as well.

We detected the pan-macrophage marker CD68 in the deep stroma layer, and the iron englobed macrophages below the epithelium (Fig. 2A, CD68). Immunohistochemical staining for CD11c (M1 macrophage marker), CD163, and CD206 (M2 macrophage markers) markers revealed the presence of $\mathrm{CD} 11 \mathrm{c}^{+}, \mathrm{CD}_{163^{+}}$, and $\mathrm{CD} 206^{+}$cells immediately just below the OE epithelium, and only M2 macrophages in the deep layer of the stroma (Fig. 2A, region within the dotted line). Double staining for iron and CD163 revealed the presence of M2 macrophages englobed iron ions in the deep layer of the OE stroma (Fig. 2A arrow). We located significantly more $\mathrm{CD} 63^{+}$and $\mathrm{CD} 206^{+}$cells, than $\mathrm{CD} 11 \mathrm{c}^{+}$cells in the deep layer of OE (Fig. 2C, $\mathrm{P}<0.001$ ). CD68 $8^{+}$cells, along with conspicuous staining of $\mathrm{CD} 63^{+}$and $\mathrm{CD} 206^{+}$cells, were observed in the stroma of CCC tumors (Fig. 2B). We also observed macro- phages with englobed iron (Fig. 2B arrow) and far more abundant M2 than M1 macrophages in CCC specimens (Fig. 2D, $\mathrm{P}<0.001$ ). There were no statistically significant differences in the numbers of cells positive for CD11c, CD163, and CD206 between OE and CCC (data not shown).

Endometrial epithelium infiltrates the stroma of $O E$. We postulated that the macrophages with englobed iron in the deep layer of the OE stroma resulted from endometrial epithelium infiltration of the OE stroma. We further hypothesized that M2 macrophages colonized the epithelium and invaded the stroma.

The endometrial epithelium forms rows in typical $\mathrm{OE}$ (Fig. 3A). Fig. 2 demonstrates more abundant M2 than M1 macrophages in the deep layers of the stroma (Fig. 3A; CD68, CD11c, CD163, and CD206 are shown in Fig. 2A). The positive rate of $\mathrm{Ki} 67$ (tumor growth marker) was low in the endometrial epithelium (4.6\% \pm 1.6 , Fig. 4A).

Staining for the epithelial cell marker keratin revealed the regions where the endometrium epithelium had infiltrated the stroma and had formed lumens in one OE specimen (Fig. 3B: Patient no. 1, region within the black square in the $\mathrm{H} \& \mathrm{E}$ staining figure) and in two CCC specimens with $\mathrm{OE}$ adjacent to CCC (Fig. 3C-D: Patient no.2-3, region within the black square in the $H \& E$ staining figure). These regions contained more abundant $\mathrm{CD}_{163}{ }^{+}$and $\mathrm{CD} 206^{+}$cells than $\mathrm{CD} 11 \mathrm{c}^{+}$cells (Figs. 3B-D and $\mathrm{S} 1$, and $\mathrm{Ki} 67$ positive rate of $14.3-38.5 \%$; Patient no.1: $38.5 \pm 6.0 \%$, Patient no. 2: $36.1 \pm 3.7 \%$, Patient no. 3: $14.3 \pm 2.4 \%$ was observed in the endometrium epithelium infiltrating the stroma (Fig. 4B-D). Endometrium, as well as ovarian follicles, stained positive for keratin. To avoid misinterpreting these findings as normal, we distinguished endometrial epithelium from ovarian follicles using CD10, a diagnostic marker of endometriosis (19). The endometrial stroma was positive, while the follicular epithelium was negative for CD10 (Fig. S2). 

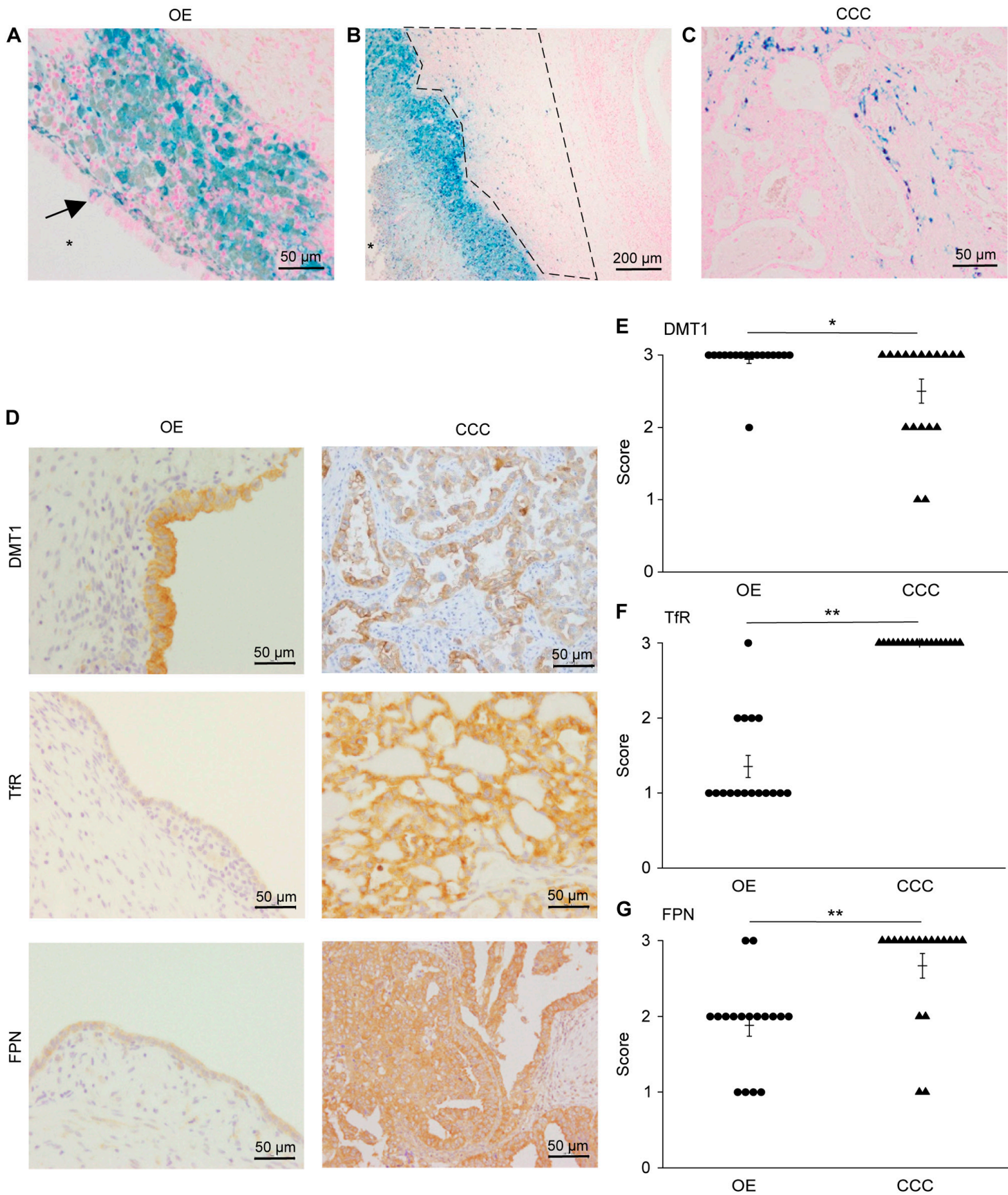

Figure 1. Iron deposits detected by Prussian blue staining and expression of iron transport proteins in the endometrial epithelium of OE and ovarian CCC tumor cells. (A) Iron deposits in the endometrial epithelium of $\mathrm{OE}$ (arrow). Macrophages englobed with iron are seen immediately below the epithelium. Magnification, $\mathrm{x} 400$. Scale bar, $50 \mu \mathrm{m}$. The star $\left(^{*}\right)$ indicates the lumen of OE. (B) Deep layer represents stroma beneath aggregated englobed macrophages (region within the dotted line). Iron deposits observed in englobed macrophages and deep layer. Magnification, x100. Scale bar, $200 \mu \mathrm{m}$. The star $(*)$ indicates the lumen of OE. (C) Iron deposits in tumor cells and stroma of CCC. Magnification, x400. Scale bar, $50 \mu \mathrm{m}$. (D) Immunolocalization of DMT1, TfR and FPN. Magnification, $\mathrm{x} 400$. Scale bar, $50 \mu \mathrm{m}$. Comparison of (E) DMT1, (F) TfR and (G) FPN expression. " $\mathrm{P}<0.05$ and ${ }^{* * *} \mathrm{P}<0.001$ via Wilcoxon rank sum test. CCC, clear cell adenocarcinoma; OE, ovarian endometrioma; DMT1, divalent metal transporter 1; TfR, transferrin receptor; FPN, ferroportin.

\section{Discussion}

In the present study, we detected iron transporters in OE and CCC using immunohistochemistry and identified M2 macrophages englobed with iron throughout the stroma of OE. The endometrial epithelium infiltrating the stroma was highly proliferative.
Although the precise mechanisms associated with the carcinogenesis of EAOC remain under investigation, the co-occurrence of the AT-rich interaction domain 1A (ARID1A) and the phosphatidylinositol-4, 5-bisphosphate 3-kinase catalytic subunit alpha (PIK3CA) mutation promotes CCC growth (20-23). Moreover, an iron-rich environment contributes to cell proliferation in $\mathrm{OE}$, with oxidative stress and reactive 

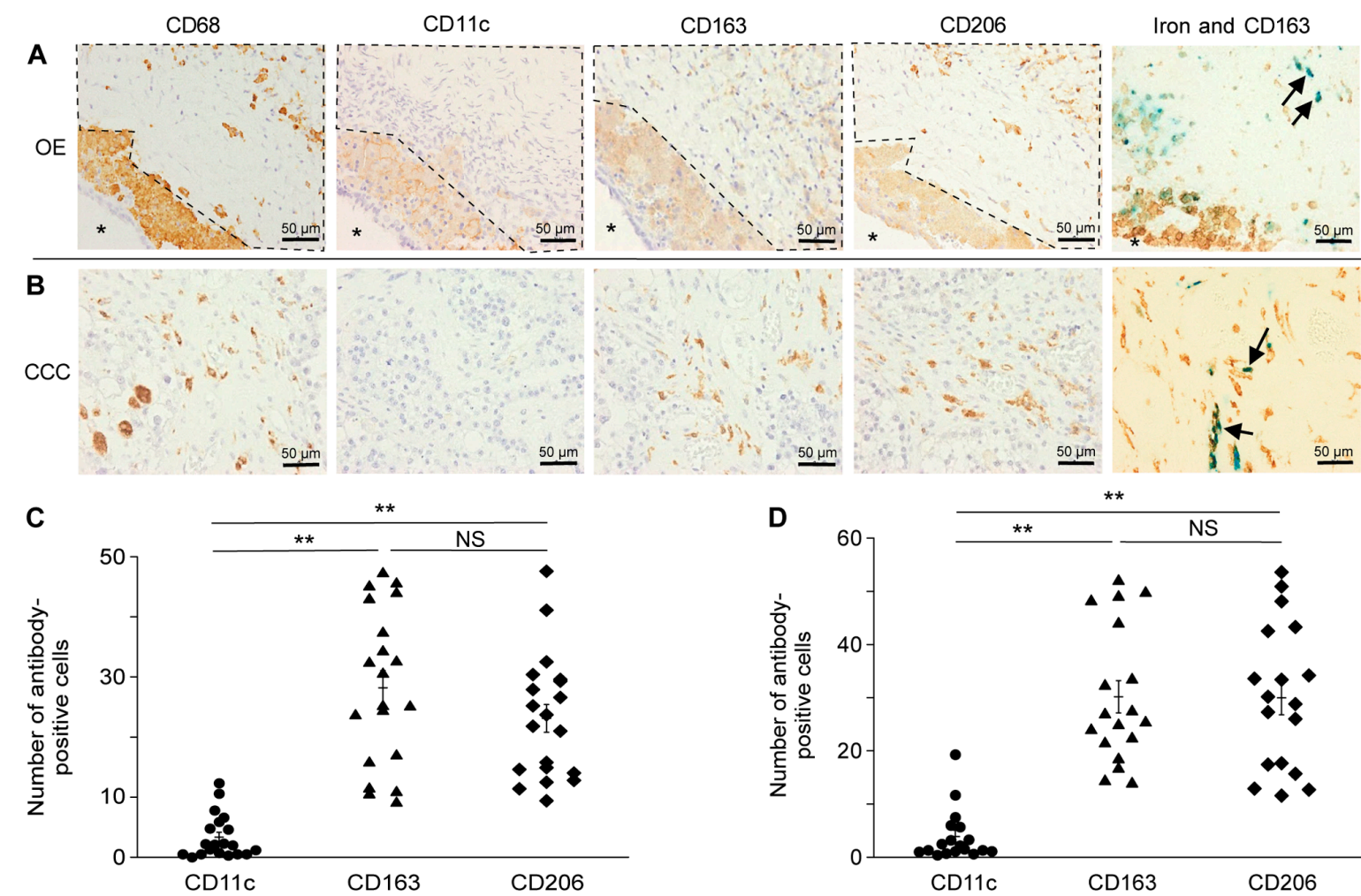

$*$ *

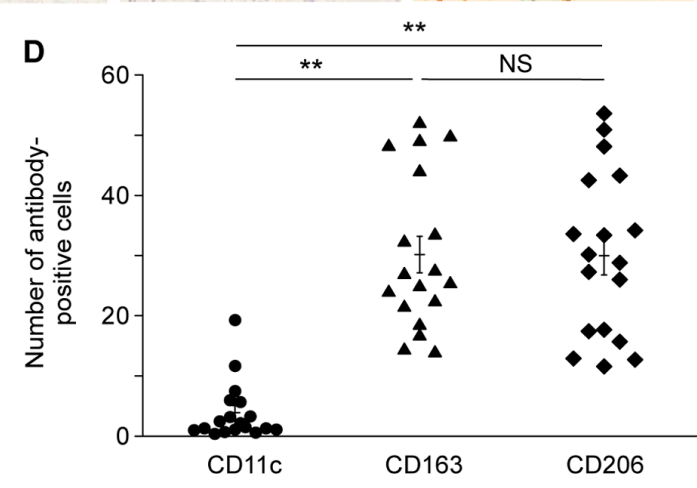

Figure 2. Immunolocalization of CD68, CD11c, CD163 and CD206. (A) Stroma under macrophages englobed with iron in OE specimens designated as deep layer (region within the dotted line). Distribution of CD68, CD11c, CD163 and CD206 in the deep layer. Double staining for iron and CD163 shows M2 macrophages englobed with iron in the stroma (arrows). The star $(*)$ indicates the lumen of OE. Magnification, x400. Scale bar, $50 \mu \mathrm{m}$. (B) Distribution of CD68, CD11c, CD163 and CD206 in ovarian CCC specimens. Double staining for iron and CD163 shows M2 macrophages englobed with iron in the stroma of CCC (arrows). Magnification, $\mathrm{x} 400$. Scale bar, $50 \mu \mathrm{m}$. Comparison of numbers of antibody-positive cells in (C) OE and (D) CCC. ${ }^{* *} \mathrm{P}<0.001$ via Steel-Dwass test (used after Kruskal-Wallis test). NS, not significant; CCC, clear cell adenocarcinoma; OE, ovarian endometrioma.

oxygen species implicated in DNA damage $(8,10,23,24)$. Thus, these findings suggest a relationship between iron and OE tumorigenesis. However, the mechanisms through which endometrial epithelium and tumor cells transport iron ions have remained unknown. Therefore, we aimed to demonstrate iron flow using immunohistochemistry. The transmembrane protein for ferric metal iron, DMT1, plays a major role in the intestinal uptake of iron ions (11). This protein is located not only on the cell surface but also in endosomes (12), with TfR also located on the cell surface. Because transferrin-binding trivalent iron molecules bind TfR, the complex becomes internalized in cells via endocytosis (12). Alternatively, FPN is the only transporter that exports iron ions in humans (25). The iron-responsive element (IRE) and iron regulatory proteins regulate DMT1, TfR, and FPN expression $(26,27)$. Hence, iron metabolism is strictly controlled by these mechanisms. This control system collapses in cancer, and the iron overload associated with cancer is largely due to iron dysregulation (28). Specifically, DMT1 expression is upregulated in colorectal cancer as compared to healthy colons (29). Furthermore, the expression of TfR and FPN is high and low in high-grade serous ovarian carcinoma, respectively, implying storage of excess iron ions in cells within this carcinoma type (30). The present findings indicate the upregulation of DMT1 and downregulation of TfR and FPN expression in OE. Moreover, the levels of the iron transporters differed in CCC tissues. We also verified that iron ions flow in and out of the endometrial epithelium, indicating that the differential abundance of trans- porters is associated with intercellular iron concentrations, which participate in carcinogenesis.

Excessive iron was detected not only in the endometrial epithelium but also in the stroma of OE, and M2 macrophages englobed iron ions in the stroma. Macrophages polarized toward the M2 phenotype generally produce anti-inflammatory cytokines and participate in tissue repair and angiogenesis (15), whereas M1 macrophages induce inflammatory cytokines and bactericidal activity. Therefore, M2 macrophages are regarded as TAMs (15). These TAMs have been detected within ascites from patients with ovarian cancer, where interaction between tumor cells and M2 macrophages induced tumor progression via signal transducer and activator of transcription 3 (STAT3) (31). In agreement with these findings, we detected more M2 than M1 macrophages in the stroma of OE and CCC. Furthermore, we identified M2 macrophages englobed with iron in the deep layer of OE stroma, which became a central focus of the study. Considering that ovarian cancer is an epithelial cancer, these M2 macrophages englobed with iron likely influence epithelial carcinogenesis by infiltrating endometrial epithelium in the stroma. The endometrial epithelium OE typically forms smooth rows of cells $(7,32)$. However, we demonstrate that endometrial epithelium infiltrates and forms lumens within the stroma. Moreover, the infiltrating epithelium was highly positive for Ki67 compared with normal endometrial epithelium. This indicates that the endometrial epithelium has potent proliferative capacity, which is a state closer to cancer. 

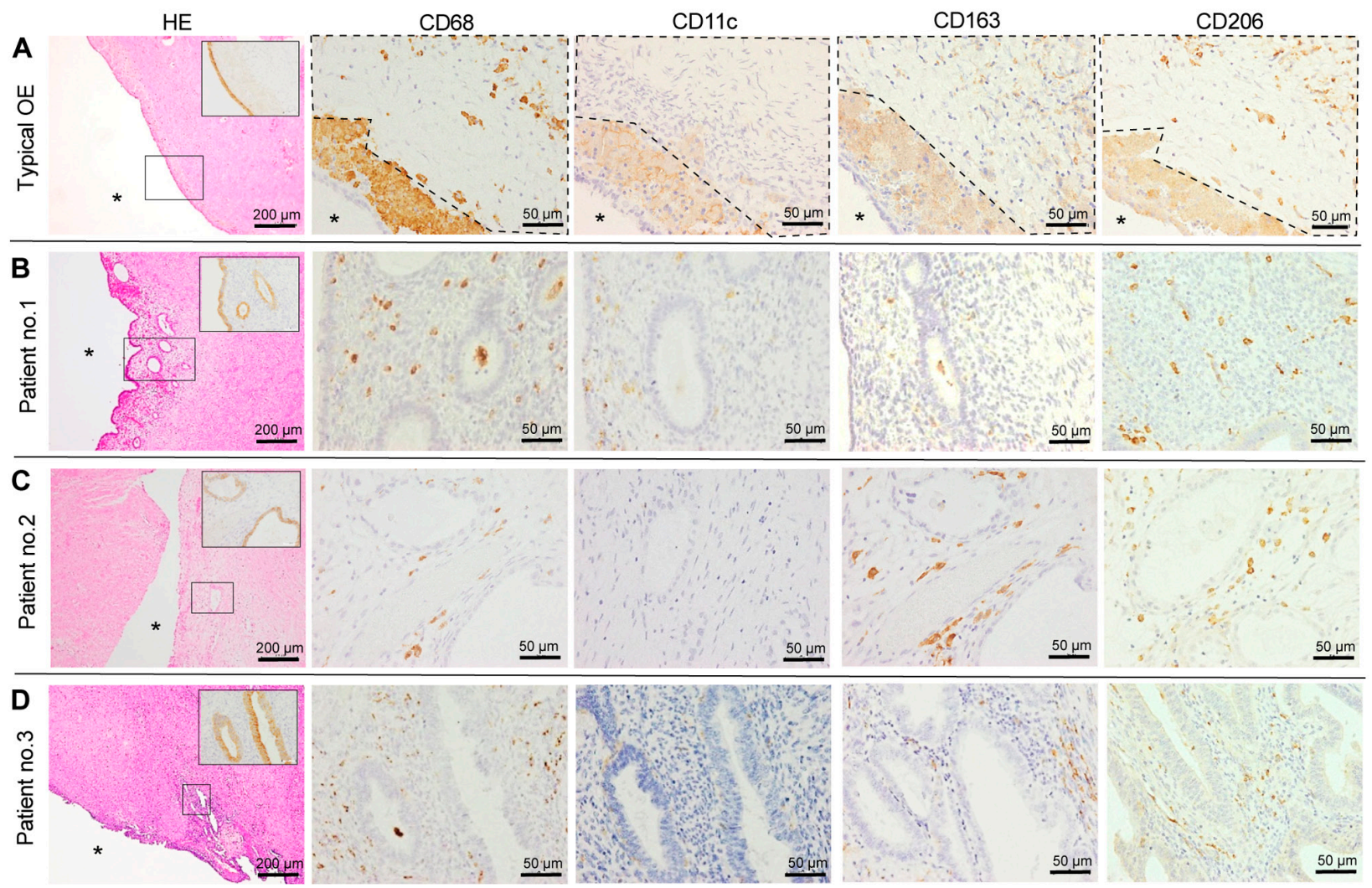

Figure 3. Keratin staining indicates endometrial epithelium infiltration of the stroma. (A) Endometrial epithelium of typical OE was used as a control. Endometrial epithelium formed a row and was stained with keratin (region within the black square in the H\&E-stained figure; magnification, $\mathrm{x} 400$ ). CD68 ${ }^{+}$,

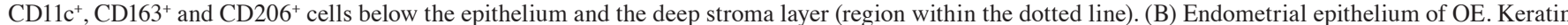
staining in the area where endometrial epithelium infiltrated stroma and formed lumens (region within the black square in the H\&E-stained figure; magnification, $\mathrm{x} 400$ ). $\mathrm{CD}^{+} 8^{+}$cells around infiltrated epithelium. CD163 and CD206 were stained more intensely than CD11c. (C and D) Endometrial epithelium adjacent to ovarian CCC from two patients. Keratin staining in the area where endometrial epithelium infiltrated the stroma and formed lumens (region within the black square in H\&E-stained figure; magnification, $\mathrm{x} 400$ ). $\mathrm{CD} 68^{+}$cells surrounded infiltrated epithelium. CD163 and CD206 were stained more intensely than CD11c. H\&E staining magnification, x100; scale bar, $200 \mu \mathrm{m}$. Other images magnification, x400; scale bar, $50 \mu \mathrm{m}$. The star $(*)$ indicates the lumen of OE. CCC, clear cell adenocarcinoma; OE, ovarian endometrioma; H\&E, hematoxylin-eosin.

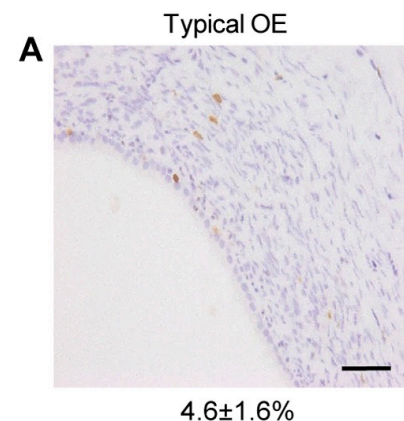

$4.6 \pm 1.6 \%$

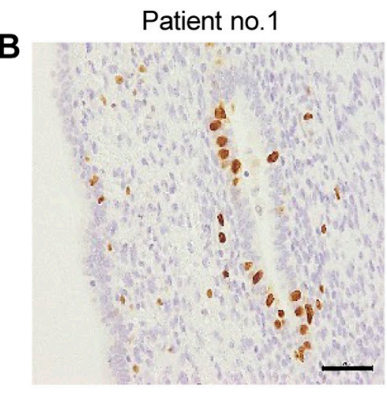

$38.5 \pm 6.0 \%$

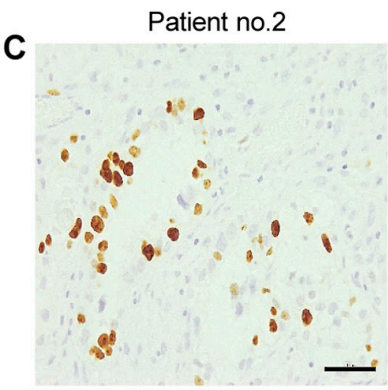

$36.1 \pm 3.7 \%$

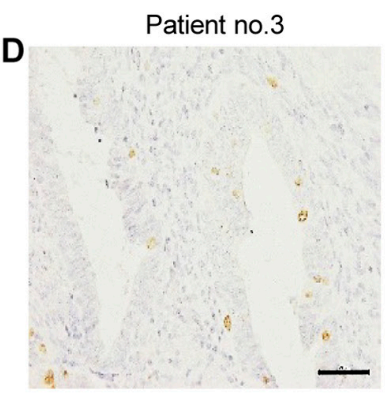

$14.3 \pm 2.4 \%$

Figure 4. Ki67 rate of endometrial epithelium infiltrating stroma. (A) Ki67 $7^{+}$rate of the typical OE. (B) $\mathrm{Ki}^{+} 7^{+}$rate of infiltrated epithelium in one OE specimen. (C and D) Ki67 rates of infiltrative epithelium in two CCC specimens with OE adjacent to CCC. Magnification, x400. Scale bar, $50 \mu \mathrm{m}$. CCC, clear cell adenocarcinoma; OE, ovarian endometrioma.

The infiltrative endometrial epithelium adjacent to CCC is likely to be a precancerous lesion. However, infiltrative endometrial epithelium in $\mathrm{OE}$ also has potential carcinogenic capacity, as benign $\mathrm{OE}$ has loss-of-function mutations in ARID1A like in CCC $(33,34)$. Considering that OE harbors ARID1A loss-of-function mutations, M2 macrophages in these regions might accelerate carcinogenesis of the endometrial epithelium infiltrating the stroma. We suggest that $\mathrm{OE}$ carcinogenesis occurs not only in endometrial cysts but also in epithelium infiltrating the stroma. Studies in a larger patient cohort are needed to verify these findings. In a preliminary study, we performed an experiment using an ovarian clear cell adenocarcinoma cell line and identified that TfR and DMT1 could be detected by western blotting. We thought that we should conduct additional experiments using ovarian endometrioma, ovarian clear cell adenocarcinoma, and normal ovary tissues as the study's primary aim was to compare between ovarian endometrioma and ovarian clear 
cell adenocarcinoma. Due to the lack of ovarian endometrioma and normal ovary cell lines, we intend further explore the role of iron transporters using an animal model, where we will be able to access more tissue samples and perform western blots. Nevertheless, our results provide insights into a potential underlying mechanism of carcinogenesis from OE to CCC while elucidating tumor growth features. Confirming these findings using pathological specimens, we could recognize the possibility of them being precancerous lesions. Moreover, careful follow-up is needed for the prevention and early detection of CCC. Further investigations of the mechanisms responsible for the infiltration of the endometrial epithelium into the stroma are warranted.

In summary, the present study discovered iron transport proteins in the epithelium of OE and CCC tumor cells. We also revealed M2 macrophages englobed with iron in the stroma of $\mathrm{OE}$ and CCC. Epithelium invading the stroma of OE implies carcinogenesis under the influence of M2 macrophages englobed with iron. These findings offer a new perspective on the malignant transformation of OE into EAOC, which may serve as a precancerous index factor.

\section{Acknowledgements}

Not applicable.

\section{Funding}

No funding was received.

\section{Availability of data and materials}

The datasets used and/or analyzed during the current study are available from the corresponding author on reasonable request.

\section{Authors' contributions}

KA, YN and HO contributed to study design. KA, YN, TT and $\mathrm{HO}$ developed the methodology and assessed the authenticity of the data. KA, YN and TT validated the data and performed formal analysis. YN and TT provided resources. KA wrote the original draft. KA, YN, TT and HO reviewed and edited the manuscript. HO supervised the study. All authors read and approved the final manuscript.

\section{Ethics approval and consent to participate}

The study was approved by the Institutional Review Board of Tokyo Women's Medical University Hospital (approval no. 5371; Tokyo, Japan). Opt-out informed consent was obtained from all patients.

\section{Patient consent for publication}

Not applicable.

\section{Competing interests}

The authors declare that they have no competing interests.

\section{References}

1. Giudice LC and Kao LC: Endometriosis. Lancet 364: 1789-1799, 2004.

2. Rogers PA, D'Hooghe TM, Fazleabas A, Gargett CE, Giudice LC, Montgomery GW, Rombauts L, Salamonsen LA and Zondervan KT: Priorities for endometriosis research: Recommendations from an international consensus workshop. Reprod Sci 16: 335-346, 2009.

3. Pearce CL, Templeman C, Rossing MA, Lee A, Near AM, Webb PM, Nagle CM, Doherty JA, Cushing-Haugen KL, Wicklund KG, et al: Association between endometriosis and risk of histological subtypes of ovarian cancer: A pooled analysis of case-control studies. Lancet Oncol 13: 385-394, 2012.

4. Fukunaga M, Nomura K, Ishikawa E and Ushigome S: Ovarian atypical endometriosis: Its close association with malignant epithelial tumours. Histopathology 30: 249-255, 1997.

5. Machida H, Matsuo K, Yamagami W, Ebina Y, Kobayashi Y, Tabata T, Kanauchi M, Nagase S, Enomoto T and Mikami M: Trends and characteristics of epithelial ovarian cancer in Japan between 2002 and 2015: A JSGO-JSOG joint study. Gynecol Oncol 153: 589-596, 2019.

6. Hooper G: The diagnosis and treatment of endometriosis. Can Med Assoc J 42: 243-246, 1940.

7. Clement PB: The pathology of endometriosis: A survey of the many faces of a common disease emphasizing diagnostic pitfalls and unusual and newly appreciated aspects. Adv Anat Pathol 14: 241-260, 2007

8. Yamaguchi K, Mandai M, Toyokuni S, Hamanishi J, Higuchi T, Takakura K and Fujii S: Contents of endometriotic cysts, especially the high concentration of free iron, are a possible cause of carcinogenesis in the cysts through the iron-induced persistent oxidative stress. Clin Cancer Res 14: 32-40, 2008.

9. Fujimoto Y, Imanaka S, Yamada Y, Ogawa K, Ito F, Kawahara N, Yoshimoto $\mathrm{C}$ and Kobayashi $\mathrm{H}$ : Comparison of redox parameters in ovarian endometrioma and its malignant transformation. Oncol Lett 16: 5257-5264, 2018.

10. Greenshields AL, Shepherd TG and Hoskin DW: Contribution of reactive oxygen species to ovarian cancer cell growth arrest and killing by the anti-malarial drug artesunate. Mol Carcinog 56: 75-93, 2017.

11. Gunshin H, Mackenzie B, Berger UV, Gunshin Y, Romero MF, Boron WF, Nussberger S, Gollan JL and Hediger MA: Cloning and characterization of a mammalian proton-coupled metal-ion transporter. Nature 388: 482-488, 1997.

12. Tabuchi M, Yoshimori T, Yamaguchi K, Yoshida T and Kishi F: Human NRAMP2/DMT1, which mediates iron transport across endosomal membranes, is localized to late endosomes and lysosomes in HEp-2 cells. J Biol Chem 275: 22220-22228, 2000 .

13. Ross SL, Tran L, Winters A, Lee KJ, Plewa C, Foltz I, King C, Miranda LP, Allen J, Beckman H, et al: Molecular mechanism of hepcidin-mediated ferroportin internalization requires ferroportin lysines, not tyrosines or JAK-STAT. Cell Metab 15: 905-917, 2012.

14. Gammella E, Buratti P, Cairo G and Recalcati S: The transferrin receptor: The cellular iron gate. Metallomics 9: 1367-1375, 2017.

15. Mantovani A, Sozzani S, Locati M, Allavena P and Sica A: Macrophage polarization: Tumor-associated macrophages as a paradigm for polarized M2 mononuclear phagocytes. Trends Immunol 23: 549-555, 2002.

16. Pollard JW: Tumour-educated macrophages promote tumour progression and metastasis. Nat Rev Cancer 4: 71-78, 2004.

17. Yamada Y, Uchiyama T, Ito F, Kawahara N, Ogawa K, Obayashi C and Kobayashi H: Clinical significance of M2 macrophages expressing heme oxygenase-1 in malignant transformation of ovarian endometrioma. Pathol Res Pract 215: 639-643, 2019.

18. Canet B, Pons C, Espinosa I and Prat J: CDC42-positive macrophages may prevent malignant transformation of ovarian endometriosis. Hum Pathol 43: 720-725, 2012.

19. Toki T, Shimizu M, Takagi Y, Ashida T and Konishi I: CD10 is a marker for normal and neoplastic endometrial stromal cells. Int J Gynecol Pathol 21: 41-47, 2002.

20. Chandler RL, Damrauer JS, Raab JR, Schisler JC, Wilkerson MD, Didion JP, Starmer J, Serber D, Yee D, Xiong J, et al: Coexistent ARID1A-PIK3CA mutations promote ovarian clear-cell tumorigenesis through pro-tumorigenic inflammatory cytokine signalling. Nat Commun 6: 6118, 2015. 
21. Yamamoto S, Tsuda H, Takano M, Tamai S and Matsubara O Loss of ARID1A protein expression occurs as an early event in ovarian clear-cell carcinoma development and frequently coexists with PIK3CA mutations. Mod Pathol 25: 615-624, 2012.

22. Samartzis EP, Noske A, Dedes KJ, Fink D and Imesch P: ARID1A mutations and PI3K/AKT pathway alterations in endometriosis and endometriosis-associated ovarian carcinomas. Int J Mol Sci 14: 18824-18849, 2013.

23. Huang HN, Lin MC, Huang WC, Chiang YC and Kuo KT: Loss of ARID1A expression and its relationship with PI3K-Akt pathway alterations and ZNF217 amplification in ovarian clear cell carcinoma. Mod Pathol 27: 983-990, 2014.

24. Ray PD, Huang BW and Tsuji Y: Reactive oxygen species (ROS) homeostasis and redox regulation in cellular signaling. Cell Signal 24: 981-990, 2012.

25. Donovan A, Lima CA, Pinkus JL, Pinkus GS, Zon LI, Robine S and Andrews NC: The iron exporter ferroportin/Slc40a1 is essential for iron homeostasis. Cell Metab 1: 191-200, 2005.

26. Schümann K, Moret R, Künzle H and Kühn LC: Iron regulatory protein as an endogenous sensor of iron in rat intestinal mucosa Possible implications for the regulation of iron absorption. Eur $\mathrm{J}$ Biochem 260: 362-372, 1999.

27. Miyazawa M, Bogdan AR, Hashimoto K and Tsuji Y: Regulation of transferrin receptor-1 mRNA by the interplay between IRE-binding proteins and $\mathrm{miR}-7 / \mathrm{miR}-141$ in the 3'-IRE stem-loops. RNA 24: 468-479, 2018.

28. Miller LD, Coffman LG, Chou JW, Black MA, Bergh J, D'Agostino R Jr, Torti SV and Torti FM: An iron regulatory gene signature predicts outcome in breast cancer. Cancer Res 71: 6728-6737, 2011
29. Xue X, Ramakrishnan SK, Weisz K, Triner D, Xie L, Attili D, Pant A, Győrffy B, Zhan M, Carter-Su C, et al: Iron uptake via DMT1 integrates cell cycle with JAK-STAT3 signaling to promote colorectal tumorigenesis. Cell Metab 24: 447-461, 2016.

30. Basuli D, Tesfay L, Deng Z, Paul B, Yamamoto Y, Ning G, Xian W, McKeon F, Lynch M, Crum CP, et al: Iron addiction: A novel therapeutic target in ovarian cancer. Oncogene 36: 4089-4099, 2017.

31. Takaishi K, Komohara Y, Tashiro H, Ohtake H, Nakagawa T, Katabuchi $\mathrm{H}$ and Takeya $\mathrm{M}$ : Involvement of M2-polarized macrophages in the ascites from advanced epithelial ovarian carcinoma in tumor progression via Stat 3 activation. Cancer Sci 101: 2128-2136, 2010

32. Tran-Harding K, Nair RT, Dawkins A, Ayoob A, Owen J, Deraney S, Lee JT, Stevens S and Ganesh H: Endometriosis revisited: An imaging review of the usual and unusual manifestations with pathological correlation. Clin Imaging 52: 163-171, 2018.

33. Wiegand KC, Shah SP, Al-Agha OM, Zhao Y, Tse K, Zeng T, Senz J, McConechy MK, Anglesio MS, Kalloger SE, et al: ARID1A mutations in endometriosis-associated ovarian carcinomas. N Engl J Med 363: 1532-1543, 2010.

34. Yachida N, Yoshihara K, Suda K, Nakaoka H, Ueda H, Sugino K, Yamaguchi M, Mori Y, Yamawaki K, Tamura R, et al: ARID1A protein expression is retained in ovarian endometriosis with ARID1A loss-of-function mutations: Implication for the two-hit hypothesis. Sci Rep 10: 14260, 2020. 